\title{
Research on a Weight Coefficient Cluster Covering Multi-objective Genetic Algorithm
}

\author{
Yan Cao, Niping Gao \\ School of Mechatronic Engineering \\ Xi'an Technological University \\ Xi'an, China \\ e-mail: jantonyz@163.com
}

\author{
Sen Cao \\ School of Accounting \\ Shandong Vocational College of Economics and \\ Business \\ Weifang, China \\ e-mail: wfcaosen@163.com
}

$$
x_{i}^{(L)} \leq x_{i} \leq x_{i}^{(U)}, i=1,2, \ldots v
$$

\begin{abstract}
A novel weight coefficient cluster covering genetic algorithm for multi-objective optimization is discussed. First, the principle and key technologies of the algorithm are presented, including cluster covering, weight coefficients, computing patterns, accurate decoding and fuzzy decoding. Then, its workflow is analyzed. An example is used to test the algorithm and the influence of algorithm parameters on computing results is also analyzed. The results show that the algorithm is effective. The algorithm can adopt several computing patterns. Both accurate decoding and fuzzy decoding have good astringency and diversity distribution.
\end{abstract}

Keywords- genetic algorithm; multi-objective optimization; operator; pareto solution; cluster covering.

\section{INTRODUCTION}

Genetic Algorithm [1] (GA) is a kind of random search method that refers to the evolution of biology. As a new global optimization algorithm, genetic algorithm has many notable features that include simpleness, versatility and strong robustness. It is also suitable for parallel processing and has high performance. It is one of the key technologies of modern intelligent computation. At present, the main involved fields include automatic control, planning, combinatorial optimization, machine learning, image processing, signal processing, artificial life, etc [2-3].

The problem of multi-objective optimization can come down to $\mathrm{M}$ mutually conflict objectives and $\mathrm{N}$ mutually restricted constraints. So how to solve it is a complex process. GA heredity acts on the entire population and emphasizes individual integration at the same time. Because the individuals can search a range of solutions in a parallel way, GA is the effective method to solve the multi-objective optimization problem [4-5].

\section{MATHEMATIC DESCRIPTION OF THE MULtI- OBJECTIVE OPTIMIZATION PROBLEM}

In general, the multi-objective optimization problem is composed of $\mathrm{M}$ objectives and $\mathrm{N}$ constraints as follows.

$$
\begin{aligned}
& \min / \max f_{m}(x),(m=1,2, \ldots m) \\
& \text { s.t. } \quad g_{u}(x) \leq 0(u=1, \ldots, q) \\
& \quad h_{v}(x)=0(v=1, \ldots, p)
\end{aligned}
$$

\section{A Weight Coefficient Cluster Covering Multi- obJective Genetic AlgORITHM}

In the paper, a weight coefficient cluster covering multiobjective genetic algorithm is put forward that is improved GA based on Pareto method. The algorithm is different from Pareto method in two aspects. 1) It adopts weight coefficients to compute fitness. 2) it utilizes cluster covering principle to update the population.

\section{A. ClusterCovering}

Cluster covering is different in set covering, which is a typical problem in combinatorial optimization. Cluster covering is the concept by which GA updates population data between generations in the course of evolution. First of all, an individual of old population becomes a new one (new chromosome) in new population through a series of operator operations. Then, judge whether the new population meets the population scale (popsize). If so, accomplish cluster covering and go to the next step. Otherwise, keep the new individual and return to add new ones.

\section{B. Weight Coefficient}

Weight coefficients reflect the importance degree of all the objectives. In the paper, the weight coefficient is defined as follows.

$$
i \in[0,1), \quad \sum_{k=1}^{n} i_{n}=1, \mathrm{n} \text { is the number of objective }
$$

functions.

In the algorithm, the mathematical expression of the fitness function is as below.

Max mode:

$$
\operatorname{Eval}(\text { chrom })=f_{1} \cdot i_{1}+f_{2} \cdot i_{2}+\ldots+f_{n} \cdot i_{n}
$$

Min mode:

$$
\operatorname{Eval}(\text { chrom })=\frac{1}{f_{1} \cdot i_{1}}+\frac{1}{f_{2} \cdot i_{2}}+\ldots+\frac{1}{f_{n} \cdot i_{n}}
$$

The developed algorithm have embedded the two kinds of fitness functions. 


\section{Calculation Mode}

In the algorithm, there are four different calculation modes, i.e., Max precision decoding, Max fuzzy decoding, Min precision decoding and Min fuzzy decoding. They are used to solve Max and Min multi-objective optimization problems.

\section{Precision Decoding and Fuzzy Decoding}

Precision decoding assures arguments lying in their domains. Its disadvantage is that its optimization performance is not high. The algorithm has coding accuracy input dialog box, for example, $0.1,0.01,0.001$, etc. When decoding, precision decoding direct uses the input precision value and decoding formula. Thus, the argument won't go beyond its domain. This decoding way is called precision decoding.

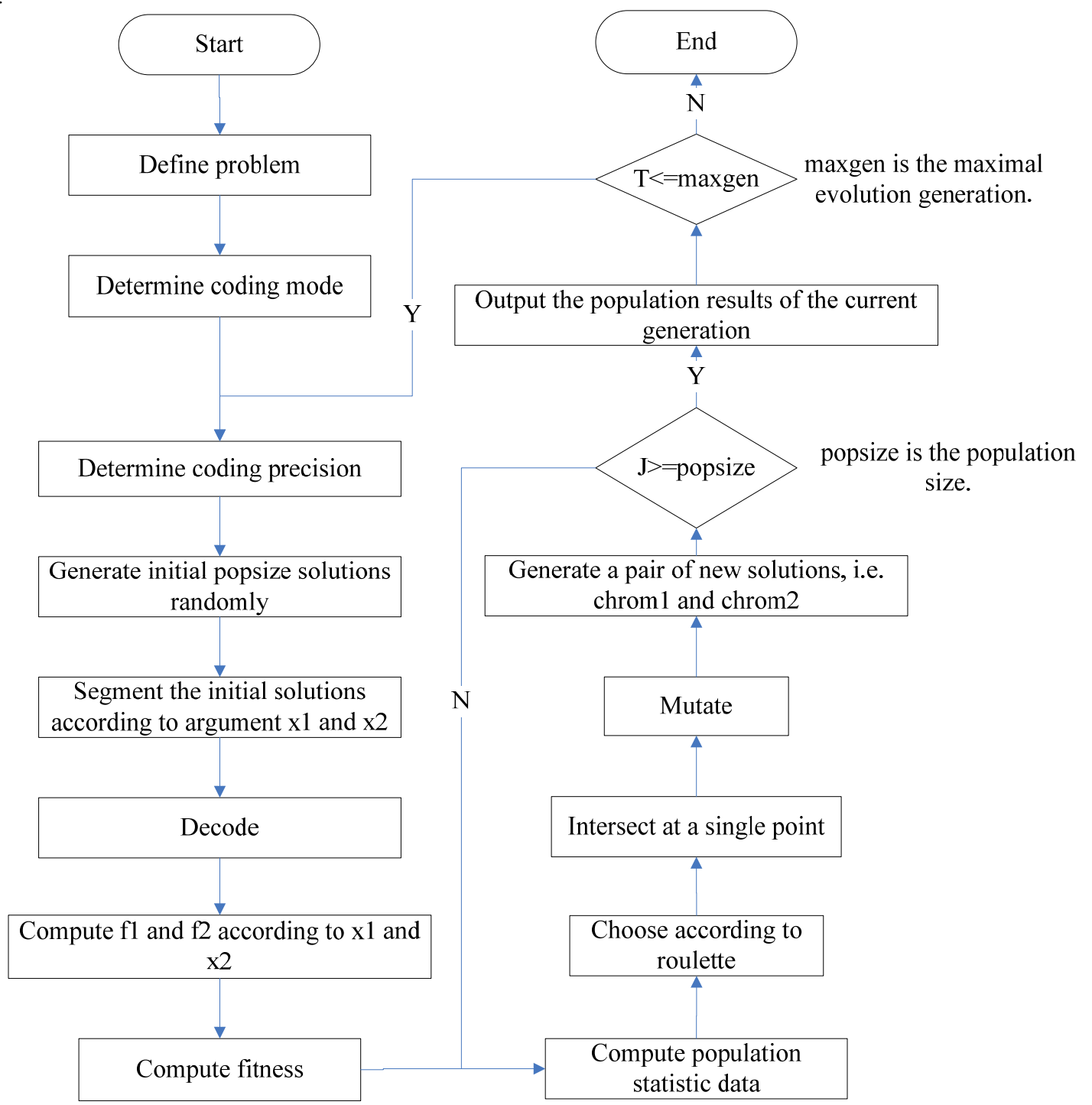

Figure 1. Weight coefficient cluster covering multi-objective genetic algorithm workflow
1) Define the problem that is the multi-objective optimization one.
Fuzzy decoding ignores the domain of the argument. Its advantage is that it can improve the optimization performance. When decoding, the argument may go beyond its domain if replacing coding accuracy $10^{-1}$ with $\delta=\frac{x_{\max }-x_{\min }}{2^{m}-1}$. It may produce unfeasible solutions. But it can greatly improve the fitness value. This decoding way is called fuzzy decoding.

\section{Workflow OF Weight COEFFiCIENT Cluster COVERING Multi-OBJeCtive GenetiC AlgORITHM}

The workflow of weight coefficient cluster covering multi-objective genetic algorithm is shown in Fig. 1.

size.
2) Determine coding scheme. The algorithm adopts binary coding. 
3) Determine coding precision $\delta$. In the paper, $\delta$ is a number $10^{-l}$, where $l$ is a precision grade.

4) Initialize a population. Generate a group of solutions. popsize is the population size.

5) Segment the individual (chromosome) into sections. Each section corresponds to an argument.

6) Decode. Convert the chromosomes into the arguments to compute the objectives in the next step. The decoding results are decimal values.

7) Use the output of 6) as input to solve the objective functions. Obtain a group of multi-objective solutions.

8) Compute fitness value. Apply Max or Min mode to calculate fitness value.

9) Compute population statistic data. They include the maximal fitness value, the minimal fitness value, average fitness value, the optimal individual, etc.

10) Choose. The algorithm adopts roulette selection. The selection probability of each individual is in direct ratio to its fitness as follows.

$$
\frac{l_{n}}{\text { eval }_{n}}=\frac{L}{\sum \text { eval }}
$$

Where, for each individual, its length on roulette is $l_{n}$, its fitness is $e v a l_{n}$, total fitness is $\sum$ eval, and the roulette perimeter is $\mathrm{L}$.

11) hybridize. The algorithm uses single-point intersection.

12) Mutate. The algorithm adopts possibility mutation point by point.

13) Produce new solutions. Choose a pair of solutions according to roulette. Produce a new pair of solutions after hybridization and mutation.
14) Judge whether a new generation is produced. In other words, judge whether popsize individuals are generated.

15) Output the results. The current population data are output as a report and curves.

16) Judge whether the stated generation number (maxgen) is reached.

17) End.

\section{EXAMPLE}

In the paper, the following example is used to verify the algorithm.

$$
\begin{gathered}
\min f_{1}=\frac{x_{1}^{2}}{4} \\
\min f_{2}=x_{1}\left(1-x_{2}\right)+5 \\
\text { s.t. } 1 \leq x_{1} \leq 4 \\
1 \leq x_{2} \leq 2
\end{gathered}
$$

The comparison of the calculation results of precision decoding and fuzzy decoding is shown in Table 1, where the precision decoding accuracy is 0.0001 .

1) The Min mode of fuzzy decoding possesses better optimization performance than that of precision decoding. When the weight coefficient 1 is 0.9 , the value of F1 adopting fuzzy decoding is less than adopting precision decoding. And when the weight coefficient 2 is 0.9 , the value

\begin{tabular}{|c|c|c|c|c|c|c|c|c|c|c|}
\hline \multicolumn{3}{|c|}{ Performance } & \multicolumn{2}{|c|}{ Weight Coefficient } & \multicolumn{2}{|c|}{$\begin{array}{l}\text { Precision Decoding } \\
\text { Precision: } 0.0001\end{array}$} & \multicolumn{2}{|c|}{$\begin{array}{l}\text { Fuzzy Decoding } \\
\text { Precision: } 0.0001\end{array}$} & \multirow{2}{*}{$\begin{array}{c}\text { Precision } \\
\text { Decoding } \\
\text { Fitness }\end{array}$} & \multirow{2}{*}{$\begin{array}{c}\text { Fuzzy } \\
\text { Decodin } \\
\text { g } \\
\text { Fitness }\end{array}$} \\
\hline $\begin{array}{l}\mathrm{C} \\
\mathrm{a} \\
\mathrm{s} \\
\mathrm{s}\end{array}$ & Objective 1 & Objective 2 & $\begin{array}{c}\text { Weight } \\
\text { coefficient } \\
1\end{array}$ & $\begin{array}{c}\text { Weight } \\
\text { coefficient } \\
2\end{array}$ & F1 & F2 & F1 & $F 2$ & & \\
\hline A & Very important & Very unimportant & 0.9 & 0.1 & 0.2542 & 4.2016 & -0.2532 & 3.3544 & 6.7510 & 7.3695 \\
\hline B & Very important & Unimportant & 0.8 & 0.2 & 0.2555 & 4.2047 & -0.2503 & 3.3614 & 6.0814 & 6.4815 \\
\hline $\mathrm{C}$ & Important & Unimportant & 0.7 & 0.3 & 0.2699 & 4.1783 & -0.4169 & 2.9077 & 6.0907 & 4.5731 \\
\hline $\mathrm{D}$ & Important & Medium & 0.6 & 0.4 & 0.3081 & 4.1037 & 1.0051 & 1.7493 & 6.0187 & 3.0873 \\
\hline $\mathrm{E}$ & Medium & Medium & 0.5 & 0.5 & 0.5771 & 3.7640 & 2.6108 & -0.2636 & 3.9969 & 7.0887 \\
\hline $\mathrm{F}$ & Medium & Important & 0.4 & 0.6 & 1.2892 & 3.1574 & 4.4308 & -1.8962 & 2.4671 & 1.4435 \\
\hline G & Unimportant & Important & 0.3 & 0.7 & 1.6280 & 2.9173 & 4.3089 & -1.7749 & 2.5372 & 1.1364 \\
\hline $\mathrm{H}$ & Unimportant & Very important & 0.2 & 0.8 & 1.7372 & 2.8424 & 3.8777 & -0.9671 & 3.3179 & 2.5819 \\
\hline I & Very unimportant & Very important & 0.1 & 0.9 & 1.7211 & 2.8549 & 2.8563 & -0.3027 & 6.1993 & 7.1717 \\
\hline
\end{tabular}
of F2 adopting fuzzy decoding is also less than adopting precision decoding. So, it is shown that fuzzy decoding is superior to precise decoding.

table I. The Comparison of CAlCulation Results BetweEn Precision Coding ANd FuzZy Coding 
2) Because fuzzy decoding may search solutions in unfeasible regions, it is likely to get the feasible solutions of higher fitness.

When the difference between weight coefficients is large, adopting fuzzy decoding can often get the solution of high fitness. But it might also lead to the destruction of constraints. Accordingly, it is needed to force the solution lying in the feasible region. If the argument is less than the minimal value of the feasible region, it takes the minimal value. If the argument is greater than the maximal value, it takes the maximal value.

\section{CONCLUSIONS}

In order to save non-inferior solutions that have high fitness value in current population in time, the weight coefficient cluster covering multi-objective genetic algorithm is studied to improve the optimal solution preservation mechanism in the paper. The algorithm adopts cluster covering technology in each generation to refresh the noninferior solutions ever and again. Thus, the final solution can approach the optimal values of sub-objectives gradually.

For a specific problem, a decision maker may not be interested in the whole Pareto optimal group, but in certain areas of the Pareto optimal group. Based on the fuzzy decoding and precision decoding of weight coefficients, once the decision maker provides the importance degree of each sub-objective, i.e., the weight coefficients, in the early computation phase, numerous solutions can be obtained. The decision maker can choose the solution for the specific problem more conveniently.
The example proves the validity of the algorithm that provides several computation modes. The calculation results show that the algorithm has good convergence and diversity. The alternate use of precision decoding and fuzzy decoding can further improve the efficiency of the algorithm. But fuzzy decoding might violate certain constraint of the algorithm.

\section{ACKNOWLEDGMENT}

The paper is supported by Special Scientific Research Project of Shaanxi 13115 Scientific and Technological Innovation Engineering Project (2010FWPT-05), Shaanxi Provincial Department of Education (09JK475), Shaanxi Major Subject Construction Project and President Scientific Research Fund of Xi'an Technological University.

\section{REFERENCES}

[1] G.. N. Xuan and R. D. Cheng, Genetic Algorithm and Engineering Optimization. Beijing, Tsinghua University Press, 2003.

[2] A. Turkcan and M. S. Akturk, "A Problem Space Genetic Algorithm in Multi-objective Optimization," Journal of Intelligent Manufacturing, vol. 14, pp. 363-378, 2003.

[3] T. Hanne, "Global Multi-objective Optimization Using Evolutionaty Algorithms," Journal of Heuristics, vol. 6, pp. 347-360, 2000.

[4] B. F. Aghi, "A Genetic Algorithm for Multiobjective Robust Design," Applied Intelligence, vol. 12, pp. 151-161, 2000

[5] R. Parsons and S. L. Canfield, "Developing Genetic Programming Techniques for the Design of Compliant Mechanisms," Digital Object Identifier, vol. 24, pp. 78-86, 2002. 